OPEN ACCESS

UWS Academic Portal

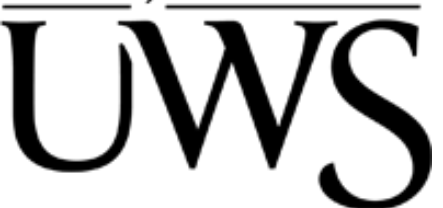

\title{
2D high-resolution crosswell seismic traveltime tomography
}

Li, Chuan; Liu, JianXin; Liao, Jianping; Hursthouse, Andrew

Published in:

Journal of Environmental \& Engineering Geophysics

DOI:

10.2113/JEEG19-003

Published: 01/03/2020

Document Version

Peer reviewed version

Link to publication on the UWS Academic Portal

Citation for published version (APA):

Li, C., Liu, J., Liao, J., \& Hursthouse, A. (2020). 2D high-resolution crosswell seismic traveltime tomography. Journal of Environmental \& Engineering Geophysics, 25(1), 47-53. https://doi.org/10.2113/JEEG19-003

\section{General rights}

Copyright and moral rights for the publications made accessible in the UWS Academic Portal are retained by the authors and/or other copyright owners and it is a condition of accessing publications that users recognise and abide by the legal requirements associated with these rights.

Take down policy

If you believe that this document breaches copyright please contact pure@uws.ac.uk providing details, and we will remove access to the work immediately and investigate your claim. 


\title{
2DHigh-resolutionCrosswell Seismic Traveltime Tomography
}

\author{
Chuan $\mathrm{Li}^{1,3}$, JianXin Liu ${ }^{1,3}$, Jianping Liao ${ }^{2,4^{*}}$, Andrew Hursthouse ${ }^{4,5}$ \\ ${ }^{1}$ School of Geoscience and Info-Physics, Central South University, Changsha, 410083, \\ China. \\ ${ }^{2}$ State Key Laboratory of Coal Resources and Safe Mining, China University of Mining \& \\ Technology, Beijing,100083, China. \\ ${ }^{3}$ Key Laboratory of Metallogenic Prediction of Nonferrous Metals and Geological \\ Environment Monitoring, Ministry of Education, Changsha 410083, China. \\ ${ }^{4}$ Hunan Provincial Key Laboratory of Shale Gas Resource Utilization, Hunan University of \\ Science and Technology, Xiangtan,411201, China. Email: 1020116@hnust.edu.cn \\ ${ }^{5}$ School of Computing, Engineering \& Physical Sciences, University of the West of Scotland, \\ Paisley PA1 2BE, UK.
}

\begin{abstract}
This paper presents a method for combining the hybrid eikonal solver and the prior velocity information to obtain high-resolution crosswell imaging. The hybrid eikonal solver in this technique can ensure rapid and reliable forward modelling of traveltime field in an unsmoothed velocity model. We also utilize the sonic well logging curve to properly develop an initial reference velocity model, and use the sonic well logging data as the prior information for the inversion part, which can restrict the problem of non-uniqueness. The results of the numerical experiment of traveltime in multi-layer media showed that the hybrid eikonal solver was more accurate than the finite difference method. The case study of an oil field in eastern China demonstrated that our method can derive a high-resolution reconstruction of the subsurface structure by inverting the primary traveltime data sets. These results suggest that even though the eikonal equation is a high frequency approximation to the wavefield, the hybrid eikonal solver can provide an accurate traveltime field in the forward modelling step of seismic crosswell tomography, which is critical to ensure high-resolution invert imaging in a highly heterogeneous environment.
\end{abstract}

\section{Introduction}

Crosswell tomography is one of the fundamental techniques in applied geophysics. Although the mathematical theory of tomography was introduced in 1917 by Radon, the first practical experiment in seismic inversion was introduced in the 1970s when Bois et al. (1972) presented a study on cross-borehole tomography from seismic traveltime dataset. Subsequently, the method was used in surveys for geophysics and engineering applications. However, in those early applications, crosswell tomography focused on providing an estimate of the sub-surface velocity structure (McMechan, 1983; Mercerat et al., 2014). As large infrastructure projects have become widely important, the use of geophysical prospecting techniques has played a key role in site investigation and regional surveys. Crosswell tomography already seems very effective in application to problems in exploration geology and environmental monitoring. Typical applications include those to characterize rock mass and provide assessment of geological features in the exploitation of mineral resources (Greenhalgh et al., 2003; Malehmir et al., 2012), to investigate aquifer and oil/gas reservoirs(Justice et al., 1989; Emery and Parra, 2013), site characterization for engineering projects (Angioni et al., 2003), monitoring $\mathrm{CO}_{2}$ injection(Harris et al., 1995; Zhang et al., 2012), characterization studies of reservoirs to provide a more accurate initial velocity model to predict precise event locations in microseismic events (Leiceaga et al., 2015), evaluation of bridge foundations (Butchibabu et al., 2017), 
estimating elastic properties and anisotropic effects in site media(von Ketelhodt et al., 2018).

The accuracy of the forward modeling in seismic traveltime computation is critical for the precision of the inversion. In the tomographic procedure, the inversion of the first arrival time data is required to recover 2-D distributions of the velocity model. In the early applications, the relatively poor quality of the crosswell tomography result was due in part to limited developments in the seismic ray theory. Bois et al. (1972) calculated the ray paths and traveltime under the Snell's law. McMechan (1983) and Justice et al. (1989) treated the sourcereceiver raypath as a straight line. Subsequently, research has focused on developing algorithms to improve the accuracy of the seismic wavefield, which can improve the precision of inversion and improve the reliability of exploration geophysics projects. Vidale (1988) presented finitedifference (referred to as FD in this paper) approximation for the calculation of travel times. Podvin and Lecomte (1991) implemented a viable alternative strategy to overcome the drawbacks of Vidale (1988) such as heterogeneity. Subsequently, Aldridge and Oldenburg (1993), used the FD operator for the first time, for rapid and accurate simulation of seismic traveltimes in crosswell tomography work. Because the FD method can rapidly obtain the traveltime field with tolerable precision, it has been widely used in seismic exploration (Liu et $a l ., 2009)$. However, the issues of the singularity at the source and accurate traveltime for the smooth velocity medium was already been clearly identified by Vidale (1988) and Podvin and Lecomte (1991).

To avoid these unwanted features and keep the accuracy in seismic traveltime field computation, i.e.ensuring the accuracy of forward modeling process in crosswell tomography, we use a new two-dimension hybrid eikonal solver that combines a spherical and plane wave operator to improve the traveltime accuracy when the computational region are close to and far away from the source (Noble et al., 2014), respectively. This hybrid FD operator can efficiently and accurately measure the traveltimes and the generation of raypaths between all sourcereceiver pairs. Moreover, Vasco and Majer (1993) pointed out that seismic wavepath tomography is more laterally continuous for layered sediments than the tomography based in the wave equation. In the inversion step, the initial velocity model is a key part of the seismic tomography(Rao et al., 2006; Kanl1, 2009). Thus, in this paper, we build a reasonable initial velocity medium based on the sonic well logging and use this prior information in the inversion step in order to get a high-resolution inversion result.

We implement hybrid FD that combines the spherical and plane wave to solve the eikonal equation in order to improve the computed efficiency and accuracy of the traveltime in the forward model. As in the inverse step, we build the initial model and constraint information from sonic well logging. We further discuss the tomography results with the initial velocity model. In addition, we invert seismic field traveltime data set from an eastern oil field in China, and the high-resolution image produced demonstrates that the inversion result can be used directly in geological interpretation.

\section{Methodology}

\section{Forward modeling: solving eikonal equation}

Using a high-frequency approximation of the elastodynamic equation, we can obtain the body waves propagation traveltime equation between the isotropic media profile of the source and receiver well, that is the eikonal equation (Vidale, 1988),

$$
\left(\frac{\partial t}{\partial x}\right)^{2}+\left(\frac{\partial t}{\partial z}\right)^{2}=\frac{1}{v^{2}(x, z)},
$$

Where $t$ is the traveltime, which is called the eikonal, $v$ is the velocity and $x, z$ represents the spatial Cartesian coordinates in horizontally and vertically, respectively. The first step to solve the eikonal eq. (1) is discretization of the velocity medium (Fig.1). Combing Vidale(1988) and Podvin and Lecomte (1991), we present 4-point and 3-point eikonal solver operator to address eq.(1). The details relating to this hybrid approach are in the following sections. 


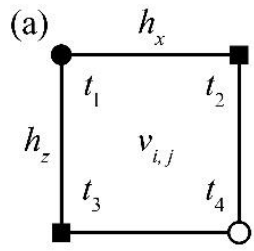

(c)

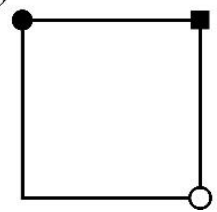

(b)

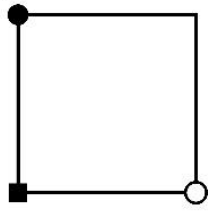

(d)

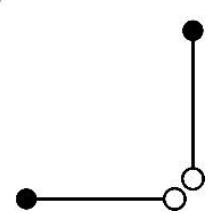

Figure 1. The discretization of the medium and FD operator. $h_{x}, h_{z}$ represent the interval step in horizontally $(i)$ and vertically $(j)$, respectively. $v_{i, j}$ stands for the velocity value in the cell. The open circle at point $(i+1, j+1)$ is solved.

\section{4-points plane wave operator}

Taking $h_{x}, h_{z}$ as the space step in the $x$ and $z$ direction (Fig.1(a)), respectively. The derivative term of the eikonal eq. (1) can be written as follows(Vidale, 1988)

$$
\begin{aligned}
& \frac{\partial t}{\partial x} \approx \frac{1}{2 h_{x}}\left(t_{1}-t_{2}+t_{3}-t_{4}\right), \\
& \frac{\partial t}{\partial z} \approx \frac{1}{2 h_{z}}\left(t_{1}-t_{3}+t_{2}-t_{4}\right) .
\end{aligned}
$$

Substituting eq.(2) into eq.(1), we can get very simple quadratic equations with one unknown in the traveltime parameter, $t_{4}$,

$$
\begin{aligned}
& \frac{1}{4}\left(\frac{1}{h_{x}^{2}}+\frac{1}{h_{z}^{2}}\right) t_{4}^{2}-\frac{1}{2}\left[\frac{\left(t_{1}-t_{2}+t_{3}\right)^{2}}{h_{x}^{2}}+\frac{\left(t_{1}+t_{2}-t_{3}\right)^{2}}{h_{z}^{2}}\right] t_{4} \\
& +\frac{1}{4}\left[\frac{\left(t_{1}-t_{2}+t_{3}\right)^{2}}{h_{x}^{2}}+\frac{\left(t_{1}+t_{2}-t_{3}\right)^{2}}{h_{z}^{2}}\right]=\frac{1}{v_{i, j}^{2}} .
\end{aligned}
$$

To insure the causality condition, we only keep the largest root of the quadratic eq.(3).

\section{3-points plane wave operator}

Because 3 points operators (Fig.1(b) and Fig.1(c)) can deal with converging wave fronts successfully. We can use it to keep the simulation accurate. The partial derivatives of eq. (1) are discrete as (Podvin and Lecomte, 1991)

$$
\frac{\partial t}{\partial z} \approx \frac{t_{1}-t_{3}}{h_{z}}, \quad \frac{\partial t}{\partial x} \approx \frac{t_{3}-t_{4}}{h_{x}} .
$$

Putting eq.(4) into eq.(1) and simplify the result leads to the 3 points plane wave operator in Fig.1(b) and Fig.1(c) are

$$
t_{4}=t_{3}+\sqrt{h_{x}^{2}\left[\frac{1}{v_{i, j}^{2}}-\frac{\left(t_{1}-t_{3}\right)^{2}}{h_{z}^{2}}\right]},
$$

and

$$
t_{4}=t_{2}+\sqrt{h_{z}^{2}\left[\frac{1}{v_{i, j}^{2}}-\frac{\left(t_{1}-t_{2}\right)^{2}}{h_{x}^{2}}\right]} .
$$




\section{1-D FD operator}

The seismic traveltime computed using 1-D FD method of the head waves (Fig. 1(d)) are written as

$$
t_{4}=t_{2}+\frac{h_{z}}{\max \left(v_{i, j}, v_{i+1, j}\right)},
$$

and

$$
t_{4}=t_{3}+\frac{h_{z}}{\max \left(v_{i, j}, v_{i, j+1}\right)} .
$$

According to Fermat's principle, seismic waves will pick the minimum time-consuming path to propagate through the medium. Thus, the minimum value of the three operators, 4 points, 3 points and 1-D FD, are the traveltime field solutions.

However, Fomel et al. (2009) point out that the plane wave FD operator is not suitable when it is close to the source. So Fomel et al. (2009) used a factored equation to address this issue. We first decompose the seismic traveltime field and the velocity in the following

$$
t=t_{0} \tau, \quad 1 / v=\alpha / v_{0} .
$$

Where $\tau$ and $\alpha$ is the perturbation of traveltime and velocity respectively. $t_{0}$ is the solution of eq. (1) for $v_{0}$, which matches

$$
\left|\nabla t_{0}\right|^{2}=1 / v_{0}^{2} .
$$

We can obtain the factored eikonal equation by substituting eq. (9) and (10) into the eikonal eq. (1)

$$
t_{0}^{2}(\nabla \tau)^{2}+2 t_{0} \tau \nabla t_{0} \nabla \tau+\tau^{2} / v_{0}^{2}=\alpha^{2} / v_{0}^{2} .
$$

Applying the same approach for the plane wave FD operator and simplify the results after substitution into eq. (1). The 4-points and 3-points spherical wave operators are in the following sections.

\section{4-points spherical wave operator}

$$
\begin{aligned}
& {\left[\frac{t_{0}^{2}}{4}\left(\frac{1}{h_{x}^{2}}+\frac{1}{h_{z}^{2}}\right)-t_{0}\left(\frac{t_{0, x}}{h_{x}}+\frac{t_{0, z}}{h_{z}}\right)+\frac{1}{v_{0}^{2}}\right] \tau^{2}+} \\
& \left\{-\frac{t_{0}^{2}}{2}\left[\frac{t_{1}-t_{2}+t_{3}}{h_{x}^{2}}+\frac{t_{1}+t_{2}-t_{3}}{h_{z}^{2}}\right]+t_{0}\left(t_{0, x} \frac{t_{1}-t_{2}+t_{3}}{h_{x}}+t_{0, z} \frac{t_{1}+t_{2}-t_{3}}{h_{z}}\right)\right\} \tau+ \\
& \frac{t_{0}^{2}}{4}\left[\frac{\left(t_{1}-t_{2}+t_{3}\right)^{2}}{h_{x}^{2}}+\frac{\left(t_{1}+t_{2}-t_{3}\right)^{2}}{h_{z}^{2}}\right]=\left(\frac{\alpha}{v_{0}}\right)^{2},
\end{aligned}
$$

where $t_{0, x}$ and $t_{0, z}$ are derived with respect to $x$ and $z$ direction respectively.

\section{3-points spherical wave operator}

The 3 points spherical wave operator in Fig.1(b) and Fig.1(c) are

$$
\begin{aligned}
& \left(\frac{t_{0}^{2}}{h_{z}^{2}}-\frac{2 t_{0} t_{0, z}}{h_{z}}+\frac{1}{v_{0}^{2}}\right) \tau^{2}+2 t_{0}\left[t_{0, x} \frac{t_{1}-t_{2}}{h_{x}}+t_{0, z} \frac{t_{2}}{h_{z}}-\frac{t_{0} t_{2}}{h_{z}^{2}}\right] \tau+ \\
& t_{0}^{2}\left[\frac{\left(t_{1}-t_{2}\right)^{2}}{h_{x}^{2}}+\frac{t_{2}^{2}}{h_{z}^{2}}\right]=\left(\frac{\alpha}{v_{0}}\right)^{2} .
\end{aligned}
$$




$$
\begin{aligned}
& \left(\frac{t_{0}^{2}}{h_{x}^{2}}-\frac{2 t_{0} t_{0, x}}{h_{x}}+\frac{1}{v_{0}^{2}}\right) \tau^{2}+2 t_{0}\left[t_{0, x} \frac{t_{1} t_{3}}{h_{x}}+t_{0, z} \frac{t_{1}-t_{3}}{h_{z}}-\frac{t_{0} t_{3}}{h_{x}^{2}}\right] \tau+ \\
& t_{0}^{2}\left[\frac{t_{3}^{2}}{h_{x}^{2}}+\frac{\left(t_{1}-t_{3}\right)^{2}}{h_{z}^{2}}\right]=\left(\frac{\alpha}{v_{0}}\right)^{2} .
\end{aligned}
$$

The seismic traveltime field will be computed using the fast sweeping approach. When we have the traveltime field of the velocity model, we can trace the rays from any receivers along the gradient of the traveltime field.

\section{D seismic first-arrival traveltime tomography}

The velocity mesh gird generated by the total of Kth rectangle cell of the velocity model $v(x$, $z$ ) (Fig.1(a)), and the slowness value of each cell, $m_{k}(\mathrm{k}=1,2, \ldots, \mathrm{K})$, is exactly the same. For this reason, the seismic ray in the cell can regard as a straight line. So, the seismic raypath between source and receiver is satisfied by a very simple equation involving distance, velocity and time. Mathematically, time $=$ distance/velocity,

$$
\Delta \mathbf{T}=\mathbf{A}(\mathbf{m}) \Delta \mathbf{m},
$$

where the matrix $\mathbf{A}(\mathbf{m})$ is the ray length of its slowness cell $\mathbf{m}$, it also be called Jacobi matrix. Because of each ray only pass through a particular cell, the $\mathbf{A}(\mathbf{m})$ is a large sparse matrix, this means that eq.(15) is ill-posed. In the inversion part, we usually set a model perturbation $\Delta \mathbf{m}$ to make $\mathbf{m}+\Delta \mathbf{m}$ approximates the true model, which associated with our selected traveltime set data. The traveltime perturbation vector $\Delta \mathbf{T}$ is in the following form

$$
\Delta \mathbf{T}=\mathbf{T}_{\text {obs }}-\mathbf{T}_{\text {calc }}(\mathbf{m}),
$$

Where $\mathbf{T}_{\text {obs }}$ is the observed traveltime from source to receiver, $\mathbf{T}_{\text {calc }}$ is the numerical traveltime calculated by hybrid FD method. Because the raypath matrix $\mathbf{A}(\mathbf{m})$ is a large, nonsquare, sparse and rank deficient, the system(15) is usually ill-conditioned and inconsistent. We therefore take the model objective function $\phi_{\mathrm{m}}$ as (Aldridge and Oldenburg 1993)

$$
\begin{aligned}
\phi_{\mathbf{m}} & =\mu_{1}^{2} \iint w_{1}(x, z)\left(\mathbf{s}-\mathbf{s}_{p}\right)^{2} \mathrm{~d} x \mathrm{~d} z+\mu_{2}^{2} \iint w_{2}(x, z)\left[\partial\left(\mathbf{s}-\mathbf{s}_{p}\right) / \partial x\right]^{2} \mathrm{~d} x \mathrm{~d} z \\
& +\mu_{3}^{2} \iint w_{3}(x, z)\left[\partial\left(\mathbf{s}-\mathbf{s}_{p}\right) / \partial z\right]^{2} \mathrm{~d} x \mathrm{~d} z .
\end{aligned}
$$

Where $\boldsymbol{s}$ and $\mathbf{s}_{p}$ are the constructed slowness model and the prior slowness model associated with the sonic logging, respectively. The weighting functions $w_{1}, w_{2}$ and $w_{3}$ are determined by the raypath at the cell, we take 1 if the ray is passing through the cell, otherwise, we take 0 . The coefficients $\mu_{1}, \mu_{2}$ and $\mu_{3}$ are the derivative constraint weight for the directional, horizontal and vertical.

The matrix form of eq. (17) can be written as follows (Aldridge and Oldenburg 1993)

$$
\boldsymbol{\phi}_{\mathbf{m}}=\sum_{n=1}^{3} \mu_{n}^{2}\left\|\mathbf{B}_{n}\left(\mathbf{m}+\Delta \mathbf{m}-\mathbf{m}_{p}\right)\right\|^{2} .
$$

Combining the $\mathrm{L}_{2}$ norm misfit function of eq. (15) and $\phi_{\mathrm{m}}$, the total objective function, $\phi(\Delta \mathbf{m})$,can be expressed as

$$
\phi(\Delta \mathbf{m})=\|\mathbf{A} \Delta \mathbf{m}-\Delta \mathbf{t}\|^{2}+\sum_{n=1}^{N} \mu_{n}^{2}\left\|\mathbf{B}_{n}\left(\mathbf{m}+\Delta \mathbf{m}-\mathbf{m}_{p}\right)\right\|^{2} .
$$

Where $N$ is the number of grid cells. Extremizing the total objective function with respect to $\Delta \mathbf{m}$, we obtain

$$
\left[\mathbf{A}^{\mathrm{T}} \mathbf{A}+\sum_{n=1}^{\mathrm{N}} \mu_{n}^{2} \mathbf{B}_{n}^{\mathrm{T}} \mathbf{B}_{n}\right] \Delta \mathbf{m}=\mathbf{A}^{\mathrm{T}} \Delta \mathbf{t}+\sum_{n=1}^{N} \mu_{n}^{2} \mathbf{B}_{n}^{\mathrm{T}} \mathbf{B}_{n}\left(\mathbf{m}_{p}-\mathbf{m}\right) .
$$

Thus, eq. (20) can be rewritten as (Aldridge and Oldenburg 1993) 


$$
\left[\begin{array}{c}
\mathbf{A} \\
\mu_{1} \mathbf{B}_{1} \\
\vdots \\
\mu_{N} \mathbf{B}_{N}
\end{array}\right] \Delta \mathbf{m}=\left[\begin{array}{c}
\Delta \mathbf{t} \\
\mu_{1} \mathbf{B}_{1}\left(\mathbf{m}_{p}-\mathbf{m}\right) \\
\vdots \\
\mu_{N} \mathbf{B}_{N}\left(\mathbf{m}_{p}-\mathbf{m}\right)
\end{array}\right]
$$

We utilize the LSQR method to solve eq.(21) to get the perturbation $\Delta \mathbf{m}$ for each iteration (Paige and Saunders, 1982).

\section{Synthetic Data Case study}

The synthetic example presented in this section is to verify the tomographic ability of our method in an unsmooth medium (Fig. 2(a)). Fig. 2(a) shows the $500 \times 500 \mathrm{~m}$ velocity model which is used to create our synthetic traveltime data set between the wells. The grid interval is $5 \mathrm{~m}$ in the horizontal and vertical direction. The background velocity of the model is $2800 \mathrm{~m} / \mathrm{s}$. There is a low $(2300 \mathrm{~m} / \mathrm{s})$ and a high $(3200 \mathrm{~m} / \mathrm{s})$ velocity layer at approximately $135 \mathrm{~m}$ and $340 \mathrm{~m}$, respectively. Moreover, we also inserted a void in the middle of the model. The sources are located at the left well, are spaced $12 \mathrm{~m}$ apart from $8 \mathrm{~m}$ to $488 \mathrm{~m}$. The traveltimes are recorded by 41 receivers (from 10 to $490 \mathrm{~m}$ with space interval of $12 \mathrm{~m}$ ) at the right well. The contour map of traveltime field and its raypath of one source at $248 \mathrm{~m}$ are presented in Fig.2(a). In the inversion part, random values have been added to the synthetic traveltimes, which follows a uniform probability distribution on $\pm 5 \mathrm{~ms}$.

The reconstructed velocity from the inversion of the crosswell traveltimes data set is shown in Fig.2(b). In twenty-five iterations, the initial root mean square (rms) traveltime misfit of $3238.5 \mathrm{~ms}$ is reduced to $75.4 \mathrm{~ms}$. Fig.2(b) illustrates that the correct position of the thin low velocity layer can be identified by the tomography method, despite its "fat" appearance. Also, Fig.2(b) can identify the cave and the high velocity layer and its position. Because the low raypath density in the cave's area, the inverted value at its center is about $120 \mathrm{~m} / \mathrm{s}$ higher.

(a)

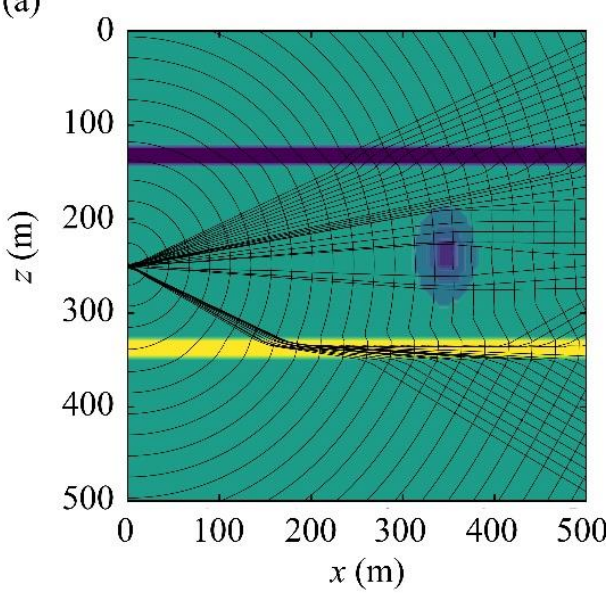

(b)

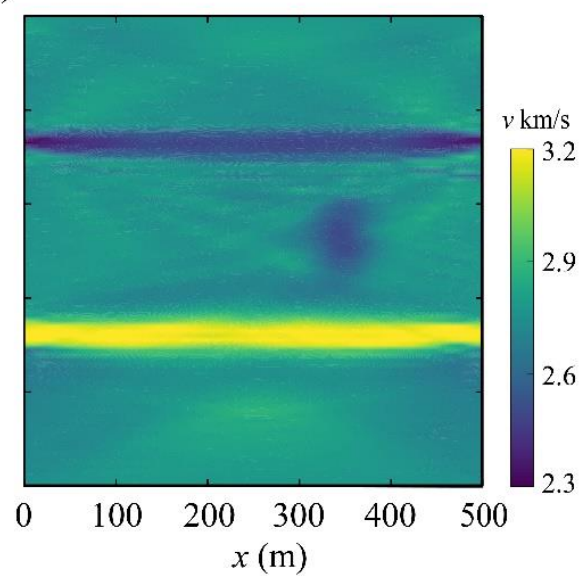

Figure 2. a) The true velocity model, the contour is the traveltime field with the source at the $248 \mathrm{~m}$, the solid line is the raypath of the source-receiver pairs. b) Reconstructed velocity image.

\section{The initial velocity model}

The solution of the inverse problem is often non-uniqueness and eq.(15) is ill-posed, so in this paper we put the velocity constraint information from sonic well logging curve into the inversion part in order to restrict the non-uniqueness and accelerate the convergence of the iterative solution (Aldridge and Oldenburg, 1993; Vasco and Majer, 1993). In this paper, we refer to the sonic logging data to build the constraint factor in the tomographic inversion procedure, that is, the minimum and maximum velocity value of the model in the inversion are 
1800 and $3800 \mathrm{~m} / \mathrm{s}$ respectively.
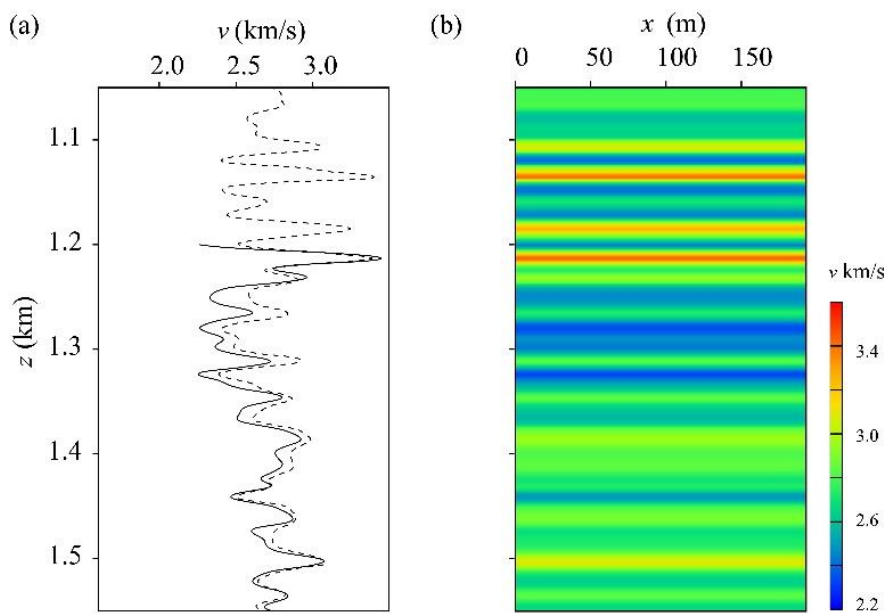

Figure 3. The sonic logging data and the initial velocity model. a) the left and right well sonic logging curve are shown with solid and dash line respectively. b) The initial velocity model based on the sonic logging.

In the inversion procedure, we pick up two types of initial velocity model. One is the uniform model that we usually use for the crosswell tomography. In this model, the value is $2800 \mathrm{~m} / \mathrm{s}$. The second is based on the sonic logging data (Fig.3(b)), in which we take the average value of the left and right-side sonic logging velocity. The grid size of the initial model in both is $2 \times 3 \mathrm{~m}$.

To test the traveltime accuracy of hybrid and FD method (Fig.4(a)). We put the source at $1350 \mathrm{~m}$ in the left well and the receivers are in the right well. Comparing the results with an analytic solution of laterally homogeneous layer models. Fig.4(b) shows that the hybrid method is close to the true solution. Figure 4 illustrates that the numerical inaccuracy of traveltimes far away from the source can be overcome by the hybrid method.

(a)

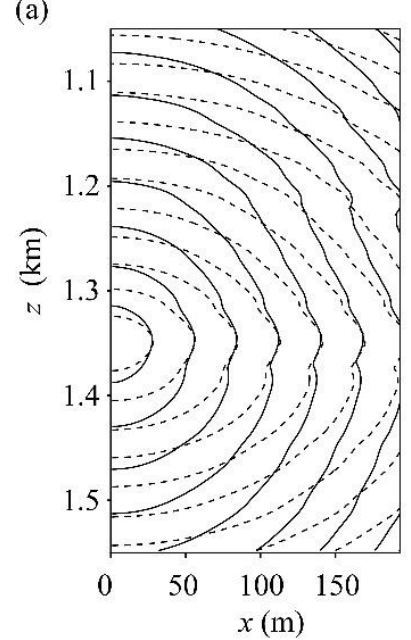

(b)

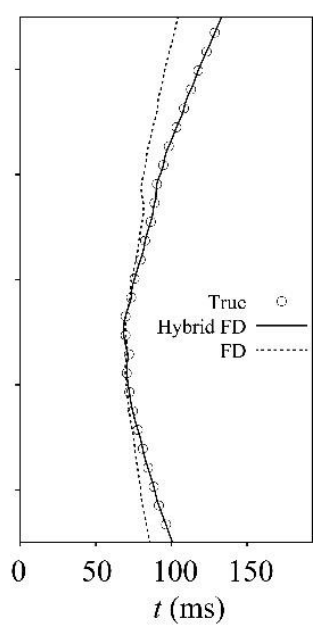

Figure 4. The traveltime field map. a) The solid and dashed line stand for the traveltime calculated by the hybrid and FD method for the initial model (Fig. 3(b)), the contour interval is $10 \mathrm{~ms}$. b) The travel time curves. The circle represents the analytical solution.

\section{Field data Example}

The 2D crosswell traveltime data set, was acquired from an oil field in eastern China. The sample rate of data is $0.5 \mathrm{~ms}$ and the total length of the seismic record is $1 \mathrm{~s}$. The offset between source and receiver well is $192.5 \mathrm{~m}$, both the receiver's interval and the shot interval are $3 \mathrm{~m}$. In 
this paper, we picked the total of 21,726 P-wave first arrival times by hand, it includes 176 shots from $1050 \mathrm{~m}$ to $1550 \mathrm{~m}$ of the well. The details of the process the raw seismic record can be found at Liao et al. (2017).The initial compressional velocity model is from sonic logging data (Fig. 3(b)), which defined in the $-5 \sim 195 \mathrm{~m}$ and $1047 \sim 1553 \mathrm{~m}$ range at horizontal and vertical region respectively. The initial model spatial discretization in the horizontal and vertical direction is $2 \mathrm{~m}$ and $3 \mathrm{~m}$,respectively. According to the sonic logging data, we also set the minimum and maximum velocity with $2100 \mathrm{~m} / \mathrm{s}$ and $3750 \mathrm{~m} / \mathrm{s}$ in the inversion procedure. In the forward part, we use the proposal hybrid FD operator with fast sweeping method to obtain the seismic traveltime field for each source. On an Intel i5 3.0 GHz processor, the elapsed CPU time for the traveltime field computation, at each iteration is $2.3 \mathrm{~s}$ with the fast sweeping method, while the fast marching method takes $7.5 \mathrm{~s}$.

The field data relates to a river deposit part of the upper member of Guantao Formation of the Miocene, and the braided river deposit is in the lower member. The deltaic facies sediment was found in the Dongying Formation of Oligocene. The thin sand-shale layer is the primary oil-bearing layer. Our aim is to detect a low velocity zone that may contain an oil and gas layer.

Figure 5 displays the first arrival traveltime tomography of the crosswell in the oil field. After 5 iterations, the image in Fig.5(a) is computed from the uniform initial velocity model and the inverted image of Fig.5(b) is from the model referred to its sonic logging data. Fig. 5(c) shows the difference of the inversion results between Fig. 5(a)and 5(b) (the velocities of Fig. 5(b) minus 5(a)), which ranges from -590 to $810 \mathrm{~m} / \mathrm{s}$. The image in Fig.5(a) at the depth around of 1200 and $1300 \mathrm{~m}$ shows that the inversion by the uniform initial model can not identify the thin low velocity. Figure 5(b) shows that putting the prior and constraint information into the inversion procedure can drive a high-resolution result for crosswell tomography. The rms misfit error at each inversion is shown in Figure 6. Compared with the image generated with the Frequency-Dependent Traveltime Tomography method (Fig. 5(d), Liao et al., 2017), these appear well matched. The thin interbedding layer at 1200 to $1250 \mathrm{~m}$ can be identified in Fig.5(b). The sand body overlap at about $1320 \mathrm{~m}$ and mudstone interlay at the approximately $1440 \mathrm{~m}$ lead to the low velocity layer. Because of some lenticular bodies can be found at $1500 \mathrm{~m}$, the velocity in this layer is relatively high.

In addition, in general, the high-frequency approximation of seismic wave equation forms the basis of eikonal equation. However, the frequency of our seismic signals in crosswell tomography is relatively low. Although eikonal solver is a convenient method for solving eikonal equation to provide us a stable and reliable travel time field, Biondi (1997), Hogan and Margrave (2007) point out that the low frequency will make the wavefront smoothing at velocity anomaly area. Maybe this is one of the reasons that cause the traditional finite difference to become invalid with the heterogeneous medium (Fig. 4), but the hybrid eikonal solver can lower the effects of low frequency (Fig. 4). As we can see in Fig. 2, the invert image can match the position of velocity anomalies zone, but the inverted result looks reasonably smooth. 


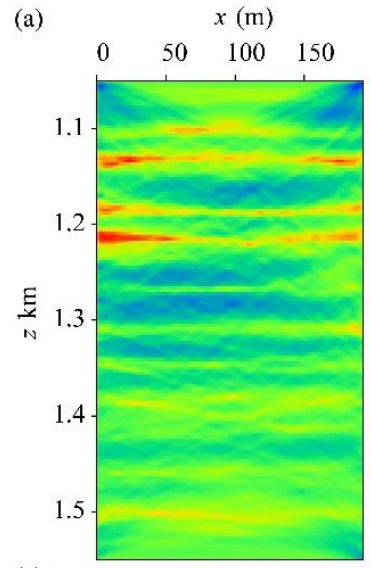

(c)

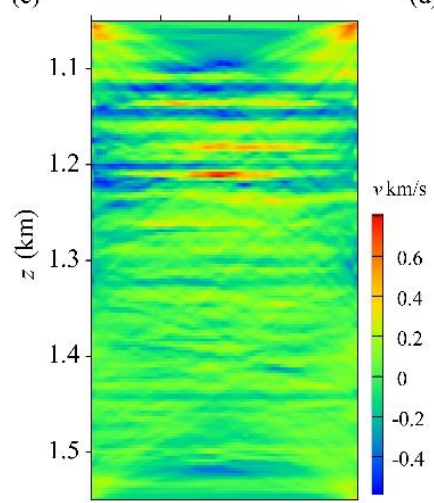

(b)

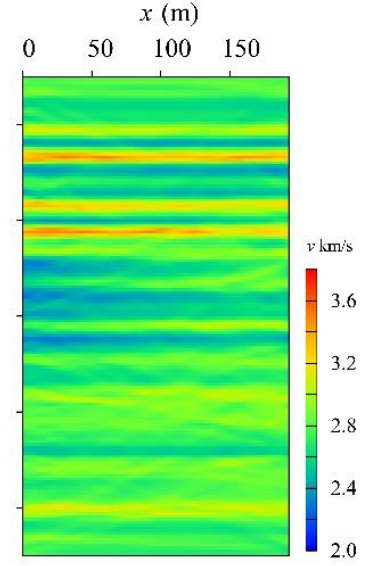

(d)

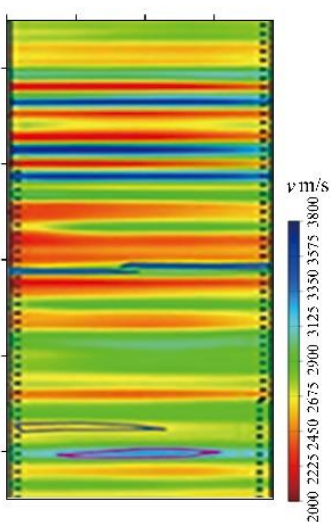

Figure 5. First arrival traveltime tomography of the interwell. The initial velocity in the inversion procedure for (a) and (b) are the based on uniform value, $2.8 \mathrm{~km} / \mathrm{s}$, and the sonic logging data (Fig. 3(b)), respectively. c) The difference of the velocities (b minus a). d) The results from Liao et al., 2017.

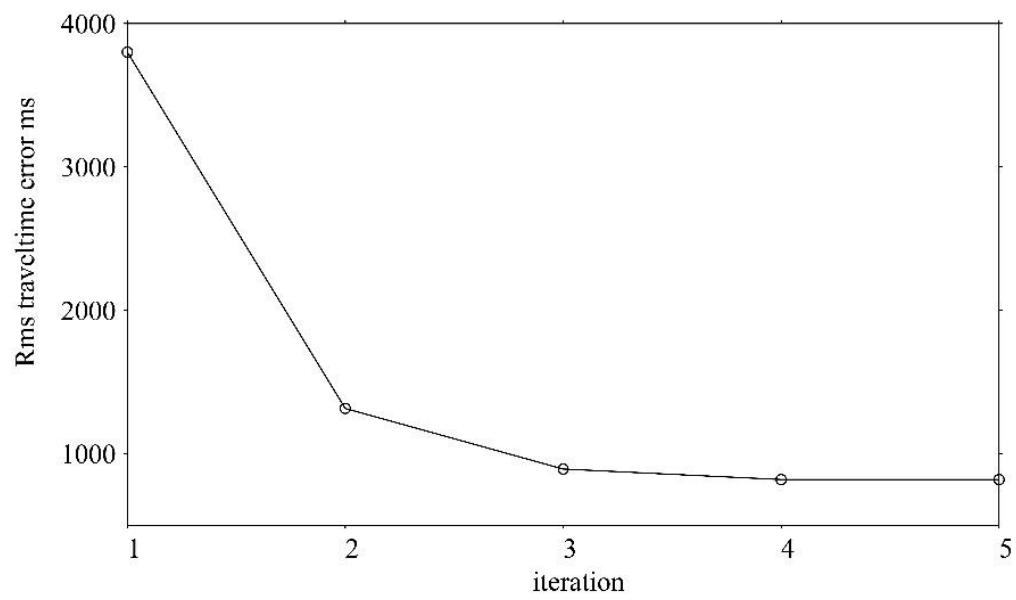

Figure 6. The rms traveltime error after each iteration of the sonic logging model (Fig.5(b)).

\section{Conclusions}

In this paper, we give a general outline of the development process for crosswell tomography and its applications in geophysical exploration. We describe in detail the hybrid eikonal solver operator to numerical the seismic traveltime field. This combines a spherical and plane approximation while close to and far away from the source respectively. The hybrid method can even provide an accurate traveltime field for the unsmooth velocity model. The hybrid method associated with the fast sweeping method can make the computation more efficient. 
Our field results have a good agreement with sonic logging data demonstrating that our method has a high enough resolution to identify the characteristics of thin layers. In the inversion procedure, the sonic logging data can help us build a true initial velocity model and provide the constraint information, which can restrict the non-uniqueness and reduce the number of iterations. Thus, using the hybrid eikonal solver method and the prior velocity data as a constraint we can provide a high-resolution image between two wells in the crosswell seismic traveltime tomography for the unsmooth environment.

\section{Acknowledgements}

This research was supported by the National Natural Science Foundation of China (41874156), State Key Laboratory for Coal Resources and Safe Mining, China University of Mining \& Technology (SKLCRSM17KFA07, SKLCRSM11KFB01), open fund of Hunan Provincial Key Laboratory of Shale Gas Resource Utilization, Hunan University of Science and Technology (E21817,E21821). Comments by editor Dr Dale Rucker, associate editor Isabel Lopes and anonymous reviewers greatly helped to clarify the text.

\section{References}

Aldridge, D.F. and Oldenburg, D.W., 1993, Two-dimensional tomographic inversion with finitedifference traveltimes: Journal of Seismic Exploration, 2, 257-274.

Angioni, T., Rechtien, R.D., Cardimona, S.J. and Luna, R., 2003, Crosshole seismic tomography and borehole logging for engineering site characterization in Sikeston, MO, USA: Tectonophysics, 368(1), 119-137.

Biondi, B., 1997, Solving the frequency-dependent eikonal equation: in Stanford Exploration Project, Report 73, 325-338.

Bois, P., Porte, M.L., Lavergne, M. and Thomas, G., 1972, WELL-TO-WELL SEISMIC MEASUREMENTS: GEOPHYSICS, 37(3), 471-480.

Butchibabu, B., Sandeep, N., Sivaram, Y.V., Jha, P.C. and Khan, P.K., 2017, Bridge pier foundation evaluation using cross-hole seismic tomographic imaging: Journal of Applied Geophysics, 144, 104114.

Emery, X. and Parra, J., 2013, Integration of crosswell seismic data for simulating porosity in a heterogeneous carbonate aquifer: Journal of Applied Geophysics, 98, 254-264.

Fomel, S., Luo, S. and Zhao, H., 2009, Fast sweeping method for the factored eikonal equation: Journal of Computational Physics, 228(17), 6440-6455.

Greenhalgh, S., Zhou, B. and Cao, S., 2003, A crosswell seismic experiment for nickel sulphide exploration: Journal of Applied Geophysics, 53(2-3), 77-89.

Harris, J.M., Nolen-Hoeksema, R.C., Langan, R.T., Schaack, M.V., Lazaratos, S.K. and James W. Rector, I., 1995, High-resolution crosswell imaging of a west Texas carbonate reservoir: Part 1—Project summary and interpretation: GEOPHYSICS, 60(3), 667-681.

Hogan CM, Margrave GM.,2007, Ray-tracing and eikonal solutions for low-frequency wavefields:inCREWES; Technical Report, 19, 1-14.

Justice, J.H., Vassiliou, A.A., Singh, S., Logel, J.D., Hansen, P.A., Hall, B.R., Hutt, P.R. and Solanki, J.J., 1989, Acoustic tomography for monitoring enhanced oil recovery: The Leading Edge, 8(2), 12-19.

Kanl1, A.I., 2009, Initial velocity model construction of seismic tomography in near-surface applications: Journal of Applied Geophysics, 67(1), 52-62.

Leiceaga, G.G., Marion, B., O'Sullivan, K.M., Bunge, G., Nielsen, J.T. and Fryer, A., 2015, Crosswell seismic applications for improved reservoir understanding: The Leading Edge, 34(4), 422-428.

Liao, J., Guo, Z., Liu, H., Dai, S., Zhao, Y., Wang, L., Wang, H. and Hursthouse, A., 2017, Application of Frequency-Dependent Traveltime Tomography to 2D Crosswell Seismic Field Data: Journal of Environmental and Engineering Geophysics, 22(4), 421-426.

Liao, J., Fu, S., Xu, Y., Li, W., Chen, J., Wang, H., Peng, S. and Hursthouse, A., 2018. Application of 3 D FKK Filtering in 3-D High-density Onshore Seismic Field Data. Journal of Environmental and Engineering Geophysics, 23(3), pp.369-376.

Liao, J., Liu, H., Li, W., Guo, Z., Wang, L., Wang, H., Peng, S. and Hursthouse, A., 2018. 3-D Butterworth Filtering for 3-D High-density Onshore Seismic Field Data. Journal of Environmental and Engineering Geophysics, 23(2), pp.223-233. 
Liu, Y., Sen, M.K. and Jackson, K.G., 2009, Advanced finite-difference methods for seismic modeling: Geohorizons.

Mackens, S., Hocine, Y. and Werban, U., 2017. Direct-push Based Seismic Crosshole Testing for Geotechnical Engineering Applications. Journal of Environmental and Engineering Geophysics, 22(3), pp.291-297.

Malehmir, A., Durrheim, R., Bellefleur, G., Urosevic, M., Juhlin, C., White, D.J., Milkereit, B. and Campbell, G., 2012, Seismic methods in mineral exploration and mine planning: A general overview of past and present case histories and a look into the future: GEOPHYSICS, 77(5), WC173-WC190.

McMechan, G.A., 1983, Seismic tomography in boreholes: Geophysical Journal International, 74(2), 601-612.

Mercerat, E.D., Nolet, G. and Zaroli, C., 2014, Cross-borehole tomography with correlation delay times: GEOPHYSICS, 79(1), R1-R12.

Noble, M., Gesret, A. and Belayouni, N., 2014, Accurate 3-D finite difference computation of traveltimes in strongly heterogeneous media: Geophysical Journal International, 199(3), 1572-1585.

Paige, C.C. and Saunders, M.A., 1982, LSQR: An Algorithm for Sparse Linear Equations and Sparse Least Squares: ACM Trans. Math. Softw., 8(1), 43-71.

Podvin, P. and Lecomte, I., 1991, Finite difference computation of traveltimes in very contrasted velocity models: a massively parallel approach and its associated tools: Geophysical Journal International, 105(1), 271-284

Rao, Y., Wang, Y. and Morgan, J.V., 2006, Crosshole seismic waveform tomography - II. Resolution analysis: Geophysical Journal International, 166(3), 1237-1248.

Vasco, D.W. and Majer, E.L., 1993, Wavepathtraveltime tomography: Geophysical Journal International, 115(3), 1055-1069.

Vidale, J., 1988, Finite-difference calculation of travel times: Bulletin of the Seismological Society of America, 78(6), 2062-2076.

von Ketelhodt, J.K., Fechner, T., Manzi, M.S. and Durrheim, R.J., 2018, Joint inversion of cross-borehole P-waves, horizontally and vertically polarized S-waves: tomographic data for hydro-geophysical site characterization: Near Surface Geophysics, 16(5), 529-542.

Zhang, F., Juhlin, C., Cosma, C., Tryggvason, A. and Pratt, R.G., 2012, Cross-well seismic waveform tomography for monitoring $\mathrm{CO} 2$ injection: a case study from the Ketzin Site, Germany: Geophysical Journal International, 189(1), 629-646. 